\title{
Assessing How the Zika Virus Induces Apoptosis and Cell Disruption in Neural Precursor Cells, and Societal Impacts of Zika: A Review
}

\author{
Meng Ji
}

\section{Introduction}

The Zika virus (ZIKV) is a Flavivirus that was first isolated in rhesus macaque monkeys in 1947 (Huang et al., 2016; Quammen, 2016). Flaviviruses are a genus of single-stranded RNA viruses that can cause common ailments such as Yellow Fever, Dengue Fever, Japanese Encephalitis, and West Nile virus (WNV) (Centers for Disease Control and Prevention [CDC], 2017). The ZIKV is a recent headline due to its associations with several neurological disorders. Upon initial infection, symptoms are mild, and can include headaches, fevers, muscle and joint pain, and rashes (Quammen, 2016; CDC, 2017). In 2015, an outbreak of Zika infection in Brazil drew researchers' attention to the virus (Huang et al., 2016). The outbreak concurred with births of babies with microcephaly, a congenital condition characterized by small head sizes. ZIKV-related microcephaly has been related to abnormal brain development, resulting in mild to extreme physical and cognitive health consequences (Huang et al., 2016).

In 2007, more than a hundred cases of ZIKV infections were found on Yap, a small island in the Southwestern Pacific. Two years later, the 
virus had found itself a new location in Northeastern Brazil. ZIKV spread rapidly in Brazil once established, and then throughout Latin America and the Caribbean (WHO, 2017; Ventura et al., 2016). Due to the perplexing number of babies born with smaller than normal heads coinciding with the rapid spread of ZIKV infection, ZIKV and microcephaly were declared public health emergencies in February of 2016 by the World Health Organization (WHO) (Huang et al., 2016).

ZIKV is primarily a concern for pregnant women, however, if the epidemic is not dealt with, sickness from infection can yield crippling wage loss for individuals and their families; the spread of the epidemic can result in economic disruption. This paper will focus on the target neural cells in the brain and how pathogenesis of ZIKV causes abnormal brain development. Moreover, the consequences of ZIKV and microcephaly in vulnerable populations will be discussed, with included recommendations for the prevention of infection. The paper will do so by primarily drawing from current literature from the microbiology and virology fields, and sociology and economics. Together, this cross-disciplinary review will bring to light the economic and societal impacts an epidemic can leave behind, and emphasize the need for improvement to public health infrastructure.

\section{What is ZIKA?}

Under the Baltimore classification system for viruses, which categorizes viruses depending on their genome type (DNA or RNA) and their method of replication, Zika is a Group IV positive single stranded RNA virus of the Flaviviridae virus family (Huang et al., 2016; CDC, 2017). ZIKV is an arbovirus, as it is transmitted by insects, specifically, the Aedes mosquito that was originally found in tropical or subtropical zones, but now exists on all continents except Antarctica (Huang et al., 2016). Members of the Aedes genus have previously played a large role in viral transmissions. Species Aedes aegypti and Aedes albopictus have been recognized to be a primary vector for ZIKV (CDC, 2017).It was not until 2007 that ZIKV became recognized as a disease causing pathogen (Posen et al., 2016). ZIKV now joins the list of viral TORCH pathogens because it disturbs the development of the embryo, where TORCH stands for toxoplasmosis, other, rubella virus, cytomegalovirus, and herpes simplex virus (Ming et al., 2016; Garcez et al., 2016). TORCH factors often compromise proper brain development in utero because these pathogens can cross the blood-placental barrier and cause congenital defects, such as microcephaly and associated, or other, cognitive disabilities (Ming et al., 2016).

ZIKV is related to many other flaviviruses such as the DENV, Japanese Encephalitis, and WNV (Huang et al., 2016; CDC, 2017). Flaviviruses are assembled of its genomic viral RNA, a lipid membrane, and three main structural proteins (capsid $(\mathrm{C})$, premembrane $(\mathrm{M})$, and envelope (E) protein), as shown in Figure 1 (Speer and Pierson, 2016). ZIKV's RNA genome encodes seven nonstructural proteins, proteins that become expressed in infected cells but not incorporated into the virion particle, and three structural proteins which are incorporated in the virion particle. The encapsulation protein is a considerable structural protein encoded by ZIKV: it encodes an envelope glycoprotein (Env) that is responsible for host cell surface recognition and binding (Speer and Pierson, 2016; Cox et al., 2015). Env binds to the host cell's endosomal membrane to initiate clathrin-mediated endocytosis. Once inside the host cell, ZIKV assembles at the endoplasmic reticulum and moves anterograde towards the Golgi Apparatus. Infected cells often secrete a nonstructural protein 1 (NS1), which aid in viral replication and infection of further cells (Speer and Pierson, 2016).

The target cells of the ZIKV appear to be the neural precursor cells (NPCs) in the brain, inducing cell death and reduced proliferation of NPCs. 


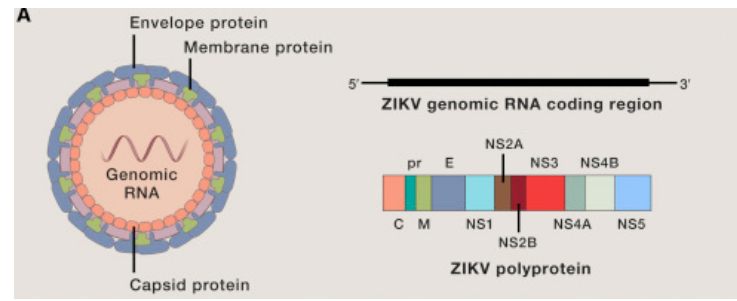

Figure 1. Zika Virus structure displaying genome and coded proteins - ZIKV genome consists of single stranded RNA. Genome encodes three structural proteins (premembrane $(\mathrm{M})$, envelope (E), capsid (C)) and seven nonstructural proteins (NS1, NS2A, NS2B, NS3, NS4A, NS4B, NS5). Includes map of ZIKV genomic coding regions (Li et al., 2016).

\section{Neural Precursor Cells (NPCs)}

Neural precursor cells (NPCs) are pluripotent stem cells that have the ability to differentiate into three main cell types that make up the human central nervous system (CNS): astrocytes, neurons, and oligodendrocytes (Ming et al., 2016). During neurogenesis, NPCs undergo either symmetric proliferative cell division or asymmetric differentiative cell division. Once NPCs perform differentiation into neurons and glial cells, they start building the neocortex of the brain, which covers the exterior of the two brain hemispheres. The neocortex makes up approximately two-thirds of the total brain mass and is involved in higher learning. Some of the neocortex functions include sensory perception, generation of motor commands, spatial reasoning, conscious thought, and language (Florio and Huttner, 2014).

\section{ZIKV-Related Microcephaly}

Microcephaly is a congenital condition characterized by a decreased brain circumference resulting in a smaller than normal head (Garcez et al., 2016; Cohen, 2016; Ventura et al., 2016). The smaller head circumference (Figure 2) is usually 2 standard deviations below the mean for the sex and age of the child (Ventural et al., 2016). When an infant is born with a head 3 standard deviations below the mean, it is con- sidered to be severe microcephaly and there is a higher risk of death. Microcephaly can occur alone or with other brain abnormalities such as vision problems, hearing loss, developmental delays, and motor problems (Cauchemez et al., 2016; CDC, 2017). Microcephaly can range from mild to severe and thus, the related problems will range from mild to severe and are often lifelong (Cauchemez et al., 2016). The CDC (2017) reports that microcephaly has a range of causes. Some babies may acquire a mutation in their genes, alcohol consumption during pregnancy can induce it, and certain infections during the mother's pregnancy may also increase the risk of microcephaly (CDC, 2017). As previously mentioned, ZIKV and microcephaly were declared public health emergencies by the WHO in early 2016, when the two coincided during the Brazil outbreak (Ventura et al., 2016). Infants with microcephaly were reported among many mothers infected with ZIKV during pregnancy. This led to the CDC (2017), among other scientists, to proclaim that there was enough evidence to conclude that ZIKV infections during pregnancy, especially if the mother becomes infected during the first trimester, may cause microcephaly, among other brain defects. The first trimester of pregnancy appears to be more vulnerable to brain malformations than the second or third trimesters, as this is when brain development is at a maximum, and the risk of brain malformations decreases as the fetus matures. Honein et al. (2017) found that in a study of 442 completed pregnancies, $6 \%$ had a baby with evidence of a ZIKV-related defect, primarily microcephaly, whereas among women infected with ZIKV during the first trimester, $11 \%$ had a child with a birth defect.

In ZIKV-related microcephaly, there is an increased level of apoptosis of NPCs and cortical progenitor cells (Garcez et al., 2016). Other researchers say that ZIKV preferentially infects and induces an apoptotic response in NPCs as well as astrocytes, oligodendrocyte precursor cells, and microglia. Retallack et al. (2016) adds that, upon infection, cell lines stemming 
from the primary NPCs demonstrate a more active apoptotic response. Because there is a deficiency of NPCs due to apoptosis, less neurons are made for the brain resulting in a smaller than normal cortex, precipitating the cognitive and physical impairments of microcephaly (Vogel, 2016). Wu et al. found that many genes that regulate the cell cycle were significantly reduced upon ZIKV infection (Nguyen et al., 2016). The cell cycle genes that were infected were frequently interrupted and led to slower cell growth. This slower cell growth could also contribute to microcephaly (Vogel, 2016). Generally, ZIKV seems to downregulate the cell cycle while upregulating apoptosis of cells of the CNS (Ming et al., 2016; Nguyen et al., 2016).

Microcephaly currently has no cure and the only treatment options depend on severity. Infants with microcephaly will often need lifelong routine check-ups to monitor their health and development (CDC, 2017). Some health complications of microcephaly were mentioned previously, but more could include facial distortions, hyperactivity, seizures, and problems with balance and coordination (Mayo Clinic Staff, 2017). Such health consequences may result in lower educational achievements and career objectives due to their shortfall of mental and physical capabilities. Furthermore, infants with microcephaly are believed to have a decreased life expectancy due to their brain impairment (Coghlan, 2016). Depending on the degree of severity, some babies will need more intensive care and treatment throughout their life to manage their health. Early intervention programs are available to support coping parents such as speech therapy, physical therapy, and seizure medications (Mayo Clinic Staff, 2017).
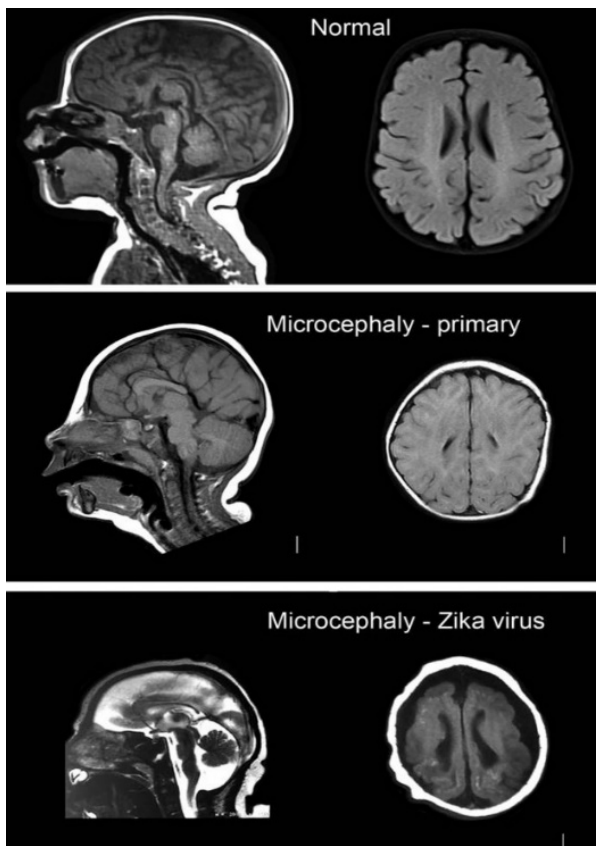

Figure 2. ZIKV infected brain causing microcephaly - Comparison of brain of a baby that has developed normally (top), brain of a baby with primary microcephaly (middle), and brain of a baby whose mother was infected with ZIKV (bottom). The bottom picture demonstrates a severe reduction in brain circumference (Kader, 2016).

\section{ZIKV Targets}

The scientific consensus on the primary targets of ZIKV are cortical NPCs while neurons are less susceptible (Li et al., 2016; Retallack et al., 2016; Tang et al., 2016). However, NPCs, radial glial cells, astrocytes, oligodendrocyte precursor cells, and microglia in humans all seem to eventually succumb to ZIKV (Retallack et al., 2016). To determine what cell types were infected in the fetal brain, Li et al. (2016) used apical and basal progenitor transcription factors that play a role in embryonic development, to verify NPCs as being the primary target in mice brain. Apical progenitor cell markers (Sox2 and Pax6), basal progenitor cell marker (Tbr2), and immature neuron markers (Tuj1 and Dcx) were 
used to identify the NPC cell type while their nuclei were stained with DAPI, after injection of ZIKV into embryonic mice brains. Sox2, Pax6, and Tbr2 were positive for infection whereas the immature neuron markers were negative. This corroborates the consensus that the ZIKV primarily affects NPCs, by suppressing the differentiation ability of apical and basal progenitor cells (Li et al., 2016; Retallack et al., 2016).

In one study, slices of cortical human brain tissue from organotypic cultures were exposed to 3 different ZIKV strains. After 3-4 months of fetus conception, high rates of viral infection can be seen in the ventricular and subventricular zones (VZ and SVZ) of the tissue culture where NPCs are primarily located. The infection was contained to mainly the VZ and SVZ with evidence of increased apoptosis in that area as NPCs become vulnerable to cell death and reduced proliferation when infected (Retallack et al., 2016). When NPCs on the VZ and SVZ are infected and undergo cell death, this gives rise to improper neurogenesis of the subgranular zone of the hippocampus, an area responsible for learning and memory (Li et al., 2016). This cell death could be a contributor of the cognitive deficiencies associated with microcephaly. Consequently, when comparing the 3 ZIKV strains, similar margins of infection against NPCs were observed across all 3 . The infected tissue culture was observed again at 4.5 months after conception: the cortical plate (CP) and subplate (SP) where mature neurons and post-mitotic cells migrate to, were observed to have a higher infection rate throughout. Glial markers GFAP and Sox2 identified a significant infection rate in astrocytes within the CP (Retallack et al., 2016). These results indicate that post-mitotic cells, such as neurons, eventually become vulnerable to infection (Li et al., 2016).

After the 2015 Zika outbreak in Brazil, the Brazilian ZIKV strain was isolated and infected into mice. Observation through transmission electron microscope showed overt signs of viral assembly in NPCs and eventually neurons $(\mathrm{Cu}-$ gola et al., 2016). The amount of viral particles rapidly increased over time in NPC and neuronal cultures, suggesting that ZIKV particles are efficient replicators (Cugola et al., 2016; Retallack et al., 2016).

\section{Cell Cycle Disruption and Cell Death}

When ZIKV infects host cells, the virus hijacks the cell's own machinery to quickly replicate its own viral DNA in order to disrupt the host's cell cycle, or slow it down, and then kill the cell (Vogel, 2016). It is suggested that ZIKV inhibits NPCs from exiting the cell cycle, and subsequent differentiation and proliferation. Infection not only leads to suspension of pluripotent stem cells in S phase, but proliferating NPCs exhibit a much longer $\mathrm{S}$ phase than those ready for differentiation. Dysregulation of the normal cell cycle is believed to be a contributing factor to microcephaly among other brain abnormalities (Boeuf et al., 2016; Li et al., 2016).

Several experiments have been conducted after the Brazilian epidemic to try and understand how ZIKV and microcephaly are related. Mice model experiments confirmed that the viral particles breached the cell cycle of NPCs, as well as slowing their progression (Boeuf et al., 2016; Huang et al., 2016). To find out the cause for microcephaly in mice, Huang et al. (2016) tested for apoptosis marker Cleaved Caspase 3 (CC3) in brain cells using antibody-CC3 for staining. Pervasive CC3 apoptosis activation was found throughout the brain, with the occipital cortex and hippocampus having a significantly higher density (Huang et al., 2016). Embryonic mice brains showed greater density of CC3 apoptosis in the intermediate zone (IP) and CP before cell death (Huang et al., 2016). In another study, ZIKV infection led to increased cell death via Caspase 3 activation in a culture of human NPCs just 3 days after initial infection (Tang et al., 2016; Cugola et al., 2016). Consequently, microcephaly and other brain abnormalities resulted as a repercussion. Death of cortical NPCs and decreased neuronal production from viral invasion are direct contributors to microcephaly, and positive staining of CC3 apoptosis showed great 
evidence that ZIKV induced this cell death pathway (Garcez et al., 2016; Huang et al., 2016).

Clear indications of abnormal brain development such as microcephaly were also observed in neurosphere models (Cugola et al., 2016; Garcez et al., 2016). A neurosphere is a plated culture system composed of floating clusters of neural stem cells from the brain area of interest. Neurospheres are commonly used to observe 3D cell growth in vitro (Jessberger et al., 2008). Mock-infected and ZIKV-infected neurospheres were generated by growing NPCs in suspension. Upon examination of the ZIKV-infected model, the neurospheres showed abnormal growth characteristics and cell detachment, and grew significantly smaller, whereas the mock-infected stem cells generated healthy, round neurospheres (Figure 3A, 3B) (Cugola et al., 2016; Garcez et al., 2016). Most of the ZIKV-infected neurospheres were apoptotic and died after a couple of days (Figure 3D). It was further observed that viral particles had become bound to the host's membranes and gained entry into the mitochondria of infected cells. Overall, this appropriately suggests that ZIKV induces apoptosis in human NPCs and impairs proper brain formation (Garcez et al., 2016).

Generally, in ZIKV-induced microcephaly, there is a pattern of NPC deficiency from the virus interrupting its cell cycle by inhibition of the cell's exit, and causing a cell death pathway, ultimately leading to less neurons produced for proper cortex development. A dire consequence as mentioned before, is microcephaly. Microcephaly patients often exhibit a significantly smaller brain, and with that comes brain impairments that affect motor, visual, hearing, and cognitive functions, depending on severity (Garcez et al., 2016).
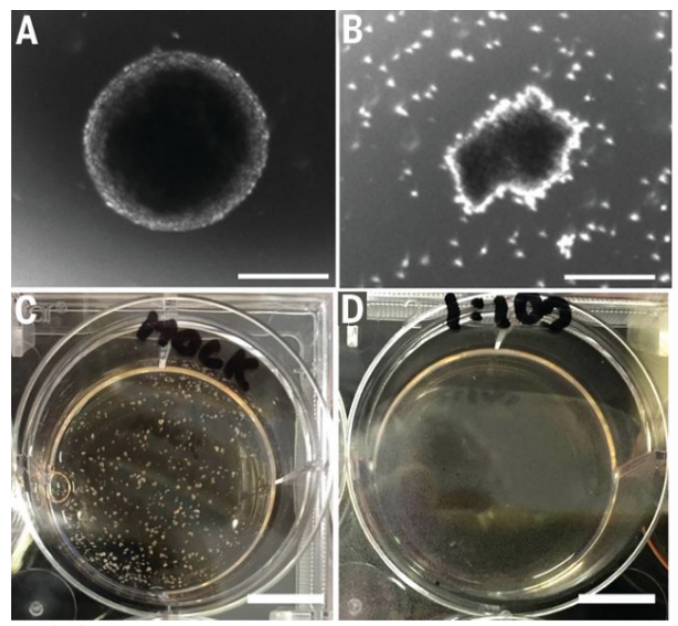

Figure 3. ZIKV alters morphology and halts growth of neurospheres - A) A control neurosphere with normal spherical morphology after 3 days in vitro $(I V)$. B) A ZIKV-infected neurosphere with abnormal cell morphology and cell detachment after 3 days $I V$. C) Mock-infected medium plate containing normal spherical neurospheres after 6 days $I V$. D) ZIKV-infected medium containing very few surviving neurospheres after 6 days $I V$ (Garcez et al., 2016).

\section{AXL Receptor}

As mentioned before, ZIKV directly infects NPC during fetal brain development to cause cell cycle dysregulation and apoptosis (Li et al., 2016). Research shows that NPCs express a protein that aids in ZIKV entry and infection. Axl, a tyrosine kinase receptor that is encoded by the Axl gene, is expressed in human NPCs (Meertens et al., 2016; Robbins \& Bender, 2016). Axl belongs to the TAM (Tyro3, Axl, Mertk) family of receptor tyrosine kinases and is involved in clearance of dead cells from apoptosis and regulation of innate immunity in humans (Meertens et al., 2016). The Axl receptor is highly abundant in the fetal cerebral cortex, where ZIKV-susceptible cells are expressed. NPCs, radial glial cells, astrocytes, microglia all express Axl in the fetal brain (Boeuf et al., 2016; Li et al., 2016; Ming et al., 2016). TAM receptors generally sustain cell growth and survival. Moreover, analysis of the 
TAM genes reveal that they play a crucial role in maintaining cortical NPCs survival, proliferation, and differentiation. For example, mice lacking in Axl and Mertk caused early cell differentiation and migration of NPCs in the SVZ. This can alter the course of cells during fetal development to result in a large array of unwanted defects (Ji et al., 2014).

Recent studies have suggested that the Axl receptor is the route of entry for ZIKV infection in humans (Richard et al., 2017). Axl has previously been identified as an entry point for DENV, a virus with similar properties to ZIKV. DENV is also an arbovirus transmitted by the Aedes mosquito genus of the flavivirus family. DENV fever has similar symptoms to ZIKV which can include muscle and joint pain, rashes, headaches and fevers, however, there seems to be no connection to microcephaly in DENV (Patterson et al., 2016). The ZIKV virion gains entrance into the host by binding Gas6 (Figure 4), a TAM ligand for Axl (Meertens et al., 2016; Ming et al., 2016). Gas6 recognizes and binds to a phosphatidylserine molecule on the viral envelope (E) protein. Gas6 then binds to its respective Axl receptor on the host. This bridges the entry of the flavivirus into the host's Axl protein. Recent studies concluded that TAM ligand Gas6 is pertinent for ZIKV infection through the Axl receptor. In one study, human microglial cells (CHME3) were treated with the viral strain ZIKVHD78. When TAM ligands were omitted from the culture, ZIKV infection levels drastically declined in CHME3 cells whereas restoration of Gas6 reinstated the high levels of ZIKV infection (Figure 5), as measured by levels of apoptosis (Meertens et al., 2016).

From here on, ZIKV enters Axl-expressing cells through clathrin-mediated endocytosis (Meertens et al., 2016; van der Schaar et al., 2008). Clathrin-mediated endocytosis (CME) is a pH-dependent pathway using clathrin-coated vesicles to internalize ligands bound to receptors on the target cell's plasma membrane (Karp, 2013). CME appears to be the route of entry used by the majority of the flaviviruses
(Fernandez-Garcia et al., 2016; van der Schaar et al., 2008). In a 2008 study, DENV particles diffused from the membrane to join a clathrincoated pit. DENV particles mature whilst moving through the cytoplasm until membrane fusion allows the viral genetic material to be released. To find out if ZIKV could enter independently of CME, Meertens et al. (2016) treated CHME3 with short interfering RNA to interfere with clathrin triskelion assembly on the vesicle. Recruitment of dynamin-2 proteins and clathrin heavy chains were inhibited in order to interfere with CME to see if ZIKV's entry was affected. After 48 hours, immunoblotting showed that ZIKV entry was strongly impaired. Likewise, treatment of cells with chemical and molecular inhibitors of CME severely incapacitated the DENV entry into mosquito cells (van der Schaar et al., 2008).

Researchers infected astrocytes in the presence of a non-activating antibody specific for the extracellular domain of Axl. The results concluded that blocking the Axl receptor appears to stall viral replication, however, interference with the internal kinase activity still allowed for astrocyte infection. This suggests that Axl's extracellular domain plays a role in infection (Retallack et al., 2016). Moreover, ZIKV binds Gas6 more efficiently than WNV or DENV and therefore, Axl is a significant factor for infection on fetal NPCs, astrocytes, and microglia. ZIKV's ability to bind Gas6 and enter through Axl may help explain why other flaviviruses cannot cross the fetal bloodstream to cause microcephaly (Richard et al., 2017). Decoy receptors for Axl have been constructed in an attempt to protect from flavivirus infection. One engineered decoy is the MYD1 receptor with a high affinity for Gas6. Results showed that MYD1 blocked ZIKV upon infection and cells such as microglia and astrocytes were protected. Further research is being conducted on Axl interference, but it is important to know that solely targeting Axl could take a toll on innate immunity (Meertens et al., 2016). Likewise, intercepting Axl has been linked to overproduction of inflammatory 
cytokines, promotion of microglia activation, and eventual death of NPCs. Therefore, blocking the Axl protein may slow down viral replication, but breach of normal protein function may have dire consequences (Nowakowski et al., 2016).

Ultimately, it is suggested that ZIKV particles enter host cells via clathrin-vesicles through tyrosine kinase receptor Axl, and are dynamin2 dependent as well as TAM ligand dependent (Meertens et al., 2016).

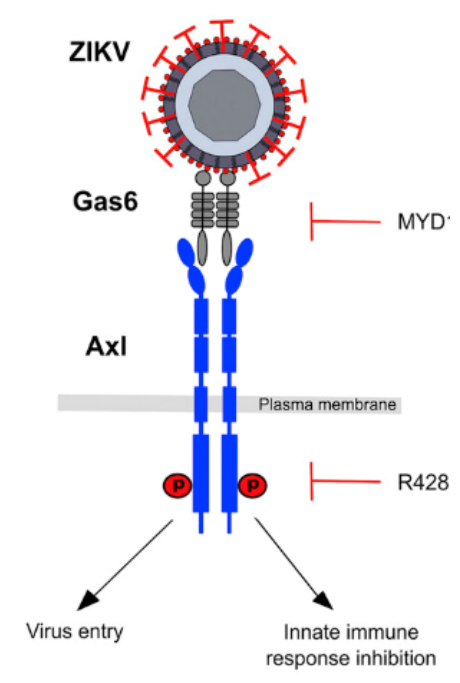

Figure 4. ZIKV binds to a TAM ligand ZIKV enters the cell by binding Gas6, an Axl ligand. Gas6 recognizes and binds to a phosphatidylserine molecule on the virus envelope (Meertens et al., 2016).

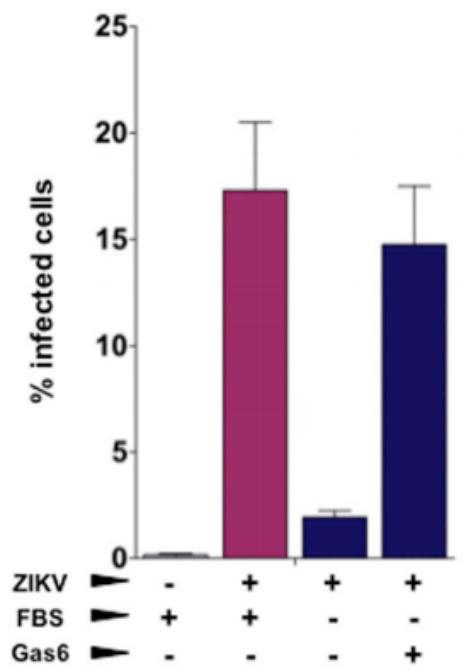

Figure 5.CHME3 cells were challenged with ZIKV in DME medium alone or containing hGas6. Pink is control. Without Gas6 as a ligand, \% of infected cells were substantially decreased, as opposed to $15 \%$ infected cells with Gas6 present.

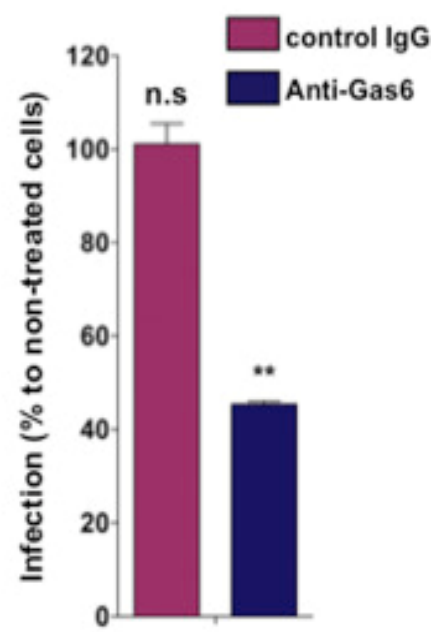

Figure 6. Astrocytes were treated with AntiGas6 ligand or control IgG and infected with ZIKV. Using anti-viral protein antibodies, it can be seen that infection \% declined when treated with Anti-Gas6 ligand. 


\section{Retinoic Acid}

A recent study suggests that microcephaly from ZIKV may not just be caused by downregulation of the cell cycle and upregulation of apoptotic genes. Retinoic acid (RA) is a small molecule that acts as an active ingredient in vitamin A. RA binds to its receptors (RAR and RXR) on certain genes' promoter regions to upregulate expression of genes that coordinate proper development of the neural tube, and other essential embryological functions. Dysregulation of RA signaling can cause brain malformations and defects, such as microcephaly (Kumar et al., 2016). For instance, previous studies where interception of RA signaling with glyphosate herbicides produced teratogenic effects on Xenopus laevis embryos, of the African aquatic frog family (Paganelli et al., 2010). RA has a significant role in building the posterior-dorsal and forebrain, therefore, disruption in the RA expression pathways from viral invasion can precipitate abnormalities in the developing brain, such as microcephaly (Kumar et al., 2016).

Since ZIKV is an RNA virus, it utilizes reverse transcriptase to synthesize a complementary DNA strand from cDNA, which is then integrated into the host's genome. Kumar et al. (2016) hypothesizes that the virus inserts repeats of a consensus sequence (5'-AGGTCA- 3') into the host's brain cells in order to disrupt the normal RA pathway. Results of the study showed that there was greater chance for microcephaly if the ZIKV strain contained the consensus sequence in its genome. Furthermore, a ZIKV strain with more sequence repeats appeared to be more fatal, causing congenital brain deformities, such as severe microcephaly. Nonetheless, the authors of the study noted that a higher number of consensus repeats could be due to a large reading frame length in the virus, and thus, further research is needed to investigate if RA signaling disruption is a common cause for brain defects.

\section{Societal and Economic Impacts of the ZIKV Epidemic}

Outbreaks of ZIKV bring a host of societal and economic burdens, and demonstrate moral failures in the community. The epidemic highlights numerous flaws in the public health infrastructure, notably in poorer countries such as Brazil. Certain demographics are more vulnerable to the virus due to the innate social and economic inequalities of the healthcare system, as well as climate change. During the H1N1 Influenza outbreak in 2009, wealthier countries received vaccines before those in immediate need because they had had preexisting contracts with manufacturing companies, even though their populations were at lower risk (Gostin and Ayala, 2017). The ZIKV costed the world billions and left its mark on the economy in many countries, especially in South America and the Caribbean (Zagorski, 2016).

The patterns of spread of ZIKV highlighted the reality that poorer families bear much of the consequences. In Brazil, families that reside in poorer parts of the country are more susceptible to ZIKV exposure and infection due to the crowded urban living conditions, insufficient and unkempt sewage systems, and pools of standing water swarming with possible infectious mosquitoes. In the United States, ZIKV has appeared in states with inadequate and expensive healthcare, where many lower-class citizens cannot afford it, or they are unable to access these basic healthcare resources (Sered, 2016). This can lead to symptoms of disease being left untreated and may possibly precipitate into further health consequences. Consequently, healthcare for millions of undocumented immigrants in the US is denied and many governors refuse to expand the insurance plan under the Affordable Care Act (Gostin and Hodge Jr., 2016). Pregnant women are especially among the neglected population of lower class citizens that cannot access basic healthcare resources. Poorer women often have longer waits for virus testing, bloodwork, maternal, and other health services. Since Brazil prohibits abortion, women are forced to 
either obtain a dangerous "back-alley" abortion, which can lead to severe injury or even death, or bear a child with the possibility of it having microcephaly, and the long-term impairments that accompany it (Gostin and Ayala, 2017; Sered, 2016). Additionally, it is believed that at least $6 \%$ of ZIKV-infected mothers will miscarry or have stillborn deliveries, whilst surviving babies will have a $13 \%$ chance of microcephaly (Gostin and Hodge Jr., 2016). Inaccessibility to appropriate healthcare for pregnant women and poorer population groups further focuses on the societal and economic inequalities that ZIKV has brought to the surface (Gostin and Ayala, 2017).

Alternatively, the increase in global temperatures enhances the vulnerability of mosquitoborne diseases, such as flaviviruses DENV, ZIKV, and WNV (Sered, 2016). The global changes in temperature, precipitation, and humidity can escalate the mosquitoes' reproduction rates and the number of blood meals they must take, and prolong their breeding season, ultimately leading to an increase in the spread of diseases they can carry. Mercer (2016) mentions that each of those climate change factors may result in more humans being exposed to mosquitoes and therefore mosquito-borne illnesses.

The World Bank estimated that the ZIKV epidemic cost the world about US $\$ 3.6$ billion in 2016. This number includes the costs of direct expenditures, productivity loss, loss from death, and the impacts of virus avoidance.

Firstly, direct expenditures are the costs to directly treat and combat the disease, such as payment for extra doctors and nurses, drugs and treatment fees, and ultrasounds of pregnant woman to monitor the development of the fetus. (Zagorski, 2016). Former United States President, Barack Obama, allocated $\$ 1.8$ billion towards response programs for the ZIKV outbreak: $\$ 828$ million was given to the CDC and $\$ 210$ million was given to the US Department of Health to address ZIKV complications (Lee et al., 2017). As of August 2016, the CDC had already spent most of its funding and was requesting additional funding, as the virus had appeared in Florida. Furthermore, an additional $\$ 81$ million was relocated from biomedical research for antipoverty and health care programs, to research of ZIKV vaccinations (Tavernise, 2016).

Productivity loss comes from the inability to work, and therefore the sickly lose out on paid work as well as contribution to the workplace (Constenla et al., 2016; Zagorski, 2016). While there is no considerable economic impact if only a handful of the population are affected by ZIKV, it is much more potent when the epidemic spreads and causes mass panic such that productivity costs can escalate. The World Bank estimates that four million people would have been infected in 2016 with roughly $20 \%$ of the four million become sick. In Latin America and the Caribbean, that would account for 750,000 people to lose out on about one week's worth of paid work (Zagorski, 2016).

Loss from death is the associated cost of infection. While the World Bank and forensic economists could not determine the value of a life, they included the costs that are necessary in order to make the society safer. Forensic economists looked to alternative studies in order to arrive at an estimate for the value of a life. For example, the US Transportation Department currently estimates that saving a life is worth over $\$ 9$ million when making decisions and rules about the safety of US roads, bridges, and tracks (Zagorski, 2016).

Lastly, disease avoidance is a large factor in the economic impact left behind by ZIKV, because the virus is active in many tourist countries, such as South America and the Caribbean. The Olympic Committee forecasted around 500,000 tourists to converge in Rio de Janeiro after it was announced that Brazil would host the 2016 Summer Olympics. However, if tourists avoided Brazil and the surrounding countries associated with the disease, there would be potential for billions of dollars to be lost without foreign fans and athletes being present (Zagorski, 
2016). There is potential for reduced tourism revenues to reach over $\$ 9$ billion across three years, or $0.06 \%$ of annual gross domestic product (GDP). In that case, the Caribbean region would require additional support from the international community to brace the economic consequences of ZIKV (Lesser and Kitron, 2016; The World Bank, 2016). Similarly, after the Chikungunya and DENV outbreak between 2006-2008, the economic impact of three Asian economies was assessed. There was a $4 \%$ decline in tourism revenues, representing a loss of about $\$ 11$ million for Gujarat, $\$ 82$ million for Malaysia, and $\$ 457$ million for Thailand (Constenla et al., 2016).

The economic impacts of disease epidemics may not always be apparent and may be significantly underestimated, especially in diseases like ZIKV because there can be lifelong effects that are manifested. Since ZIKV usually does not pose a considerable mortality risk to infected adults, it is easy to overlook the full magnitude of its impact.

\section{Impacts of ZIKV-Related Microcephaly}

While there is no vaccine available for ZIKV, most infections are asymptomatic and selflimiting, often treatable with over-the-counter medicine, hydration, and rest (Rather et al., 2017). However, infections to pregnant women can implicate microcephaly, which is probably the most dramatic consequence for ZIKVinfected newborns. Children born with microcephaly are expected to have a shorter lifespan than the average person, with the implications of microcephaly ranging from mild to extreme. The reality is that babies born with microcephaly will, in most cases, require lifelong specialized care because the underdevelopment of their brain will have a significant effect on almost all aspects of their life. As previously mentioned, microcephaly has a range of effects from vision problems and hearing loss, to physical and cognitive delays (CDC, 2017). It is predicted that children diagnosed with microcephaly may have an IQ below 70 (Cugola et al., 2016). Other possible health complications of microcephaly are speech delays, where the infant will take longer to learn and form words and sentences than that expected for their age. Speech therapy may be required in the future if this is the case. Children with microcephaly may develop difficulties with balance and coordination, therefore long-term physiotherapy may be required to assist them in swift use of their limbs (CDC, 2017). Because children with microcephaly may not reach their full cognitive and physical capacity, this puts them at a disadvantage, as they are less likely to attend school which results in lower educational achievement. Likewise, high unemployment rates have been linked to microcephalic adults (Boeuf et al., 2016). In the long run, resources of families caring for children with microcephaly will be depleted as they tend to their children's physical and developmental needs, such as glasses and hearing aids, physiotherapy costs, routine check-ups, therapists, and medication. Furthermore, the emotional toll of lifelong supervision of a child with microcephaly on the family can be demanding and devastating (CDC, 2017).

\section{Conclusion}

This review examined the recent outbreak of the Zika virus in Brazil that sparked the interests of researchers. Studies confirmed a link between ZIKV and microcephaly when the timing of the outbreak coincided with births of babies with smaller than normal heads. It was found that this flavivirus targets neural precursor cells that build the cortex of the brain. Specifically, NPCs during the first trimester seemed to be more susceptible to infection because this is the period where brain development is at a maximum. ZIKV appears to disrupt the cell cycle and induce an apoptotic response in NPCs, as can be seen in ventricular and subventricular zones of the brain. Recent studies show that a primary entry way for ZIKV is through tyrosine kinase receptor Axl, however, it is important to consider that blocking Axl can yield other severe complications. Moreover, it should be noted that recent research shows a small molecule called 
retinoic acid also being involved in infection. It is hypothesized that ZIKV inserts repeats of a consensus sequence into the host cell to disturb the normal signaling pathways of RA. Finally, it is important to consider the economic and societal impacts that an epidemic such as ZIKV has left behind. Lower class citizens usually take the brunt of disease because they lack access to appropriate resources to treat the illness. These findings emphasize the need for public health authorities to provide relevant resources and funds to vulnerable groups that face the economic and class inequalities in society. In the meantime, ZIKV infection can be prevented by being aware when travelling to places where ZIKV is active, especially if pregnant. Figure 6 shows a number of countries where the virus is active. Also, preventative measures such as wearing long sleeves and applying insect repellent are important when in mosquito-rich areas.

\begin{tabular}{lc}
\hline Location & Number of confirmed cases by September 2016 \\
\hline Brazil & 1911 \\
Colombia & 40 \\
Martinique & 12 \\
USA & 23 \\
French Polynesia & 8 \\
Panama & 5 \\
\hline
\end{tabular}

Figure 7. ZIKV related microcephaly and other congenital syndromes of the CNS.

Confirmed cases reported in 6 countries by September, 2016 (Rather et al., 2017).

\section{Limitations and Future Directions}

There are several notable limitations in this review. This review paper primarily focused on the scientific side of ZIKV pathogenesis, and its effect on neural precursor cells. More research could have been conducted on the social impacts of an infectious epidemic, especially in relation to the Western world. This paper acknowledges that the consequences of ZIKV span across many disciplines, such as politics, microbiology, and economics, and there is not one solution from one branch of knowledge. However, it does not propose specific initiatives to resolve the issue.

In order to control and prevent future disease epidemics, it is crucial to recognize that this virus (along with other arthropod-borne viruses) is spread via mosquitoes. While there currently exists no vaccine for ZIKV, education on prevention is the best way to hinder mosquito bites. Intervention strategies from public health officials to limit mosquito breeding must be implemented. For example, in poorer parts of the world where ZIKV is active, proper sewage drainage systems and adequate water management systems should be implemented to limit reservoirs where mosquitoes can reproduce (Shankar et al., 2017; Rather et al., 2017). Infected or susceptible groups should be well educated on ZIKV's mode of transmission to prevent infecting others (Shankar et al., 2017; Rather et al., 2017).

There are numerous pharmaceutical companies looking to create vaccines for the virus. Bharat Biotech in India has 2 vaccines in clinical trials. Following successful animal models, human trials should be launched to test the efficacy of vaccines. Moreover, one study showed that antibodies derived from the mother can be given to a fetus to stimulate short-term immunity. However, short-term immunity is not sufficient, and future research on discovering therapies that provide long-lasting immunity is required (Shankar et al., 2017). While ZIKV is not as big of a concern as it has been in the recent past, it is still necessary to continue surveillance for this devastating virus. 


\section{About the Author}

My name is Meng and I'm from Rockwood, Ontario! I'm studying at the University of Guelph in the Bachelor of Arts and Sciences program. My arts specialization is Criminal Justice and Public Policy and science is Microbiology. After my degree, I'm interested in going into grad school for pathology, or infectious disease, or bioremediation. My article on the Zika virus was a topic I chose with my professor, John Vessey, a neuroscientist at Guelph studying neural precursor cells. 


\section{References}

Barton, M. A., and Salvadori, M. I. (2016). Zika virus and microcephaly. CMAJ.1887,E118+. Retrieved from http://go.galegroup.com.subzero.lib.uoguelph.ca/ps/i.do?p=AONE\&sw $=\mathrm{w} \& u=g u$ e $177241 \& \mathrm{v}=2.1 \& \mathrm{it}=\mathrm{r} \& \mathrm{id}=\mathrm{GALE} \% 7 \mathrm{CA} 450039143 \& \mathrm{asid}=2 \mathrm{e} 475443 \mathrm{~b} 0 \mathrm{~d} 25 \mathrm{~b} 7 \mathrm{ab} 42 \mathrm{~b} 607 \mathrm{c} 565206 \mathrm{ed}$

Boeuf, P., Drummer, H.E., Richards, J.S., Scoullar, M.J.L., and Beeson, J.G. (2016). The global threat of Zika virus to pregnancy: epidemiology, clinical perspectives, mechanisms, and impacts.BMC Med. 14112. https://doi.org/10.1186/s12916-016-0660-0

Cauchemez, S., Besnard, M., Bompard, P., Dub, T., Guillemette-Artur, P., Eyrolle- Guignot, D., Salje, H., Van Kerkhove, M.D., Abadie, V., Garel, C., Fontanet, A., and Mallet, H-P. (2016). Association between Zika virus and microcephaly in French Polynesia, 2013-2015: a retrospective study. Lancet. 387(10033), 21 25-2132. http://doi.org/10.1016/S0140-67361600651-6

Centers for Disease Control and Prevention [CDC]. (2017). Facts about Microcephaly. CDC. [accessed June 2017]. Retrieved from https://www.cdc.gov/ncbddd/birthdefects/microcephaly.html

Coghlan, A. (2016, February 1). What will life be like for Brazil's generation of Zika babies? News Scientist. Retrieved from https://www.newscientist.com/article/2075925-what-will-life-be-likefor-brazils-generation-of-zika-babies/

Cohen, J. (2016). Zika causes microcephaly in mice. Science. DOI:10.1126/science.aaf5715

Constenla, D., de Broucker, G., and del Campo, J.M. (2016, March 7). The potential economic impact of the Zika virus. IVAC. [accessed 2017 June]. Retrieved from http://www.jhsph.edu/researc $\mathrm{h} /$ centers-and-institutes/ivac/IVACBlog

Cox, B.D., Stanton, R.A., and Schinazi, R.F. (2015). Predicting Zika virus structural biology: Challenges and opportunities for intervention. Antivir. Chem. Chemother. 24(3-4), 118-126. DOI: $10.1177 / 2040206616653873$

Cugola, F.R., Fernandes, I.R., Russo, F.B., Freitas, B.C., Dias, J.L.M., Guimarães, K.P., Benazzato, C., Almeida, N., Pignatari, G.C., Romero, S., Polonio, C.M., Cunha, I., Brandão, W.N., Ro ssato, C., Andrade, D.G., Faria, D.P, Garcez, A.T., Buchpigel, C.A., Braconi, C.T., Mendes, E., Sall, A.A., Zanotto, P.M., Peron, J.P.S., Muotri, A.R., and Beltrão-Braga, P.C.B. (2016). The Brazilian Zika virus strain causes birth defects in experimental models. Nature. 534, 267-271. DOI:10.1038/nature18296

Fernandez-Garcia, M.D., Meertens, L., Chazal, M., Hafirassou, M.L., Dejarnac, O., Zamborlini,A., Despres, P., Sauvonnet, N., Arenzana-Seisdedos, F., Jouvenet, N., and Amara, A. (2016). Vaccine and wild-type strains of Yellow Fever Virus engage distinct entry mechanisms and differentially stimulate antiviral immune responses. mBio. 71, e01956-15. DOI: 10.1128/mBio.0195615

Florio, M., and Huttner, W.B. (2014). Neural progenitors, neurogenesis and the evolution of the neocortex. Development. 141, 2182-2194. DOI:10.1242/dev.090571

Garcez, P.P., Loiola, E.C., Da Costa, R.M., Higa, L.M., Trindade, P., Delvecchio, R.,Nascimento, M.J., Brind- eiro, R., Tanuri, A., and Rehen, S.K. (2016). Zika virus impairs growth in human neurospheres and brain organoids. Science. 352(6286), 816-818. DOI:10.1126/science.aaf6116

Gostin, L. O., and Ayala, A. S. (2017). Global health security in an era of explosive pandemic potential. J. Nat'l Sec. L. E Pol'y. 91, 1-24. Retrieved from http://sfx.scholarsportal.info.subzero.lib .uoguelph.ca/guelph/docview/1869124864?accountid=11233

Gostin, L.O., and Hodge, J.G. (2016). Zika virus and global health security. Lancet Infect. Dis. 1610, 1099-1100. http://dx.doi.org/10.1016/S1473-30991630332-2. 
Honein, M.A., Dawson, A.L., Petersen, E.E., Jones, A.M., Lee, E.H., Yazdy, M.M., Ahmad, N., Macdonald, J., Evert, N., Bingham, A., Ellington, S.R., Shapiro-Mendoza, C.K., Oduyebo, T., Fine, A.D., Brown, C.M., Sommer, J.N., Gupta, J., Cavicchia, P., Slavinski, S., White, J.L., Owen, S.M., Petersen, L.R., Boyle, C., Meaney-Delman, D., and Jamieson. D.J. (2017). Birth defects among fetuses and infants of US women with evidence of possible Zika virus infection during pregnancy. JAMA. 3171, 59-68. DOI:10.1001/jama.2016.19006

Huang, W.C., Abraham, R., Shim, B.S., Choe, H., and Page, D.T. (2016). Zika virus infection during the period of maximal brain growth causes microcephaly and corticospinal neuron apoptosis in wild type mice. Sci. Rep. 6(34793), 1-8. DOI:10.1038/srep34793

Jessberger, S., Aimone, J.B., and Gage, F.H. 4.41 - Neurogenesis, In Learning and Memory: A Comprehensive Reference; ISBN 9780123705099; Academic Press: Oxford, 2008; 839858. https://doi.org/10.1016/B 978-012370509-9.00042-5.

Ji, R., Tian, S., Lu, H.J., Lu, Q., Zheng, Y., Wang, X., Ding, J., Li, Q., and Lu, Q. (2013). TAM receptors affect adult brain neurogenesis by negative regulation of microglial cell activation. $J$. Immunol. 19112, 6165-6177. DOI:10.4049/jimmunol.1302229

Kader, H. (2016, March 4). New research links Zika virus to brain defects. Seattle's Children Hospital. [accessed 2017 August]. Retrieved from http://pulse.seattlechildrens.org/new-researchlinks-zika-virus-to-brain-defects/

Karp, G. 2013. Cell and Molecular Biology: Concepts and Experiments, $7^{\text {th }}$ edition. New York, NY: John Wiley and Sons, Inc.

Kumar, A., Singh, H.N., Pareek, V., Raza, K., Dantham, S., Kumar, P., Mochan, S., and Faiq, M.A. (2016). A possible mechanism of Zika virus associated microcephaly: Imperative role of retinoic acid response element (RARE) consensus sequence repeats in the viral genome. Front. Hum. Neurosci. 10, 403. https://doi.org/10.3389/fnhum.2016.00403

Lee, B.Y., Alfaro-Murillo, J.A., Parpia, A.S., Asti, L., Wedlock, P.T., Hotez, P.J., and Galvani, A.P. (2017). The potential economic burden of Zika in the continental United States. PLoS Negl. Trop. Dis. 114, 1-20. https://doi.org/10.1371/journal.pntd.0005531

Lesser, J., and Kitron, U. (2016, July 27). The social geography of Zika. NACLA. [accessed August 2017]. Retrieved from http://nacla.org/news/2016/07/27/social-geography-zika

Li, C., Xu, D., Ye, Q., Shi, L., Qin, C.F., and Xu, Z. (2016). Zika virus disrupts neural progenitor development and leads to microcephaly in mice. Cell Stem Cell. 191, 120-126. http://dx.doi.org/10. 1016/j.st- em.2016.04.017

Li, H., Saucedo-Cuevas, L., Shresta, S., and Gleeson, J.G. (2016). The neurobiology of Zika virus. Neuron. 925, 949-958. https://doi.org/10.1016/j.neuron.2016.11.031

Mayo Clinic Staff. (2016, January 25). Microcephaly: Symptoms. Mayo Clinic. [accessed 2017 June]. Retrieved from http://www.mayoclinic.org/diseases-conditions/microcephaly/basics/symptoms/ con-2003 4823

Meertens, L., Labeau, A., Dejarnac, O., Gressens, P., Shwartz, O., and Amara, A. (2016). Axl mediates ZIKA virus entry in human glial cells and dodulates innate immune responses. Cell Stem Cell. 182, 324-333. http://dx.doi.org/10.1016/j.celrep.2016.12.045

Mercer, G. (2016 February 24). The link between Zika and climate change. The Atlantic. Retrieved from https://www.theatlantic.com/health/archive/2016/02/zika-and-climate-change/470643/

Ming, G.L., Tang, H., and Song, H. (2016). Advances in Zika virus research: Stem cell models, challenges, and opportunities. Cell Stem Cell. 196, 690-702. http://dx.doi.org/10.1016/j.stem.2016. 11.014 
Nguyen, H.N., Qian, X., Song, H., and Ming, G-l. (2016). Neural stem cells attacked by Zika virus. Cell Res. 26, 753-754. doi:10.1038/cr.2016.68

Nowakowski, T. J., Pollen, A. A., Di Lullo, E., Sandoval-Espinosa, C., Bershteyn, M., and Kriegstein, A. R. (2016). Expression analysis highlights AXL as a candidate Zika virus entry receptor in human neural stem cells. Cell Stem Cell. 185, 591-596. http://doi.org/10.1016/j.stem.2016 .03 .012

Paganelli, A., Gnazzo, V., Acosta, H., López, S.L., and Carrasco, A.E. (2010). Glyphosatebased herbicides produce teratogenic effects on vertebrates by impairing retinoic acid signaling. Chem. Res. Toxicol. 2310, 1586-1595. DOI: 10.1021/tx1001749

Patterson, J., Sammon, M., and Garg, M. (2016). Dengue, Zika and Chikungunya: Emerging arboviruses in the new world. West. J. Emerg. Med. 176, 671-679. http://doi.org/10.5811/west jem.2016.9.30904

Posen, J.H., Keystone, J.S., Gubbay, J.B., and Morris, S.K. (2016). Epidemiology of Zika virus, 1947-2007. BMJ Glob. Health. 12, e000087. DOI: 10.1136/bmjgh-2016-000087

Quammen, D. (2016, January 28). Why Zika is this year's scary virus. National Geographic. Retrieved from http://news.nationalgeographic.com/2016/01/160128-zika-virus-birth-defectsbrian-damage-history-science/

Rather, I. A., Kumar, S., Bajpai, V. K., Lim, J., and Park, Y.-H. (2017). Prevention and control strategies to counter ZIKA epidemic.Front Microbiol8305. http://doi.org/10.3389/fmicb.2017. 00305

Retallack, H., Di Lullo, E., Arias, C., Knopp, K. A., Laurie, M. T., Sandoval-Espinosa, C., Leon, W.R., Krencik, R., Ulliam, E.M., Spatazza, J., Pollen, A.A., Mandel-Brehm, C., Nowakow ski, T.J., Kreigstein, J., and DeRisi, J. L. (2016). Zika virus cell tropism in the developing human brain and inhibition by azithromycin. Proc. Natl. Acad. Sci. U.S.A.11350, 1440814413. http://doi.org/10.1073/pnas.161802 9113

Richard, A.S., Shim, B-S., Kwon, Y-C., Zhang, R., Otsuka, R., Schmitt, K., Berri, F., Diamond, M.S., and Choe, H. (2017). AXL-dependent infection of human fetal endothelial cells distinguishes Zika virus from other pathogenic flaviviruses. Proc. Natl. Acad. Sci. U.S.A. 1148, 2024-2029. DOI:10.1073/pna- s.1620558114

Robbins, H., and Bender, E. (2016, December 1). How Zika infects the growing brain. Harvard Gazette. [accessed 2017 July]. Retrieved from http://news.harvard.edu/gazette/story/2016 /12/how-zika-infects-the-growing-brain/

Sered, S. (2016, August 19). The social implications of Zika. The Hill. Retrieved from http://thehill.co m/blogs/pundits-blog/healthcare/291985-the-social-implications-of-zika

Shankar, A., Patil, A. A., and Skariyachan, S. (2017). Recent perspectives on genome, transmission, clinical manifestation, diagnosis, therapeutic strategies, vaccine developments, and challenges of zika virus research. Front Microbiol, 8. https://doi.org/10.3389/fmicb.2017.01761

Speer, S.D., and Pierson, T.C. (2016). Diagnostics for Zika virus on the horizon.Science. 353(6301), 750-751. DOI: 10.1126/science.aah6187

Tang, H., Hammack, C., Ogden, S. C., Wen, Z., Qian, X., Li, Y., Yao, B., Shin, J., Zhang, F., Lee, E.M., Christian, K.M., Didier, R.A., Jin, P., Song, H., and Ming, G. (2016). Zika virus infects human cortical neural precursors and attenuates their growth. Cell Stem Cell. 185, 587-590. http://doi.org.subzero.lib.uoguel ph.ca/10.1016/j.stem.2016.02.016 
Tavernise, S. (2016, August 30). U.S. funding for fighting Zika virus Is nearly spent, C.D.C. says. New York Times. Retrieved from https://www.nytimes.com/2016/08/31/health/us-fundingfor-fighting-zika-virus-is-nearly-spent-cdc-says.html?smid=nytcore-ipad-share\&smprod=nytcoreipad

The World Bank. (2016, February 18). World bank provides $\$ 150$ million to combat Zika virusin Latin America and the Caribbean. World Bank Group. [accessed 2017 August]. Retrieved from http://ww w.worldbank.org/en/news/press-release/2016/02/18/world-bank-provides-150-million-to-combatzika-virus-in-latin-america-and-the-caribbean

van der Schaar, H.M., Rust, M.J., Chen. C., van der Ende-Metselaar. H, Wilschut. J, Zhuang, X.,and Smit, J.M. (2008). Dissecting the cell entry pathway of Dengue virus by single-particle tracking in living cells. PLoS Pathog. 412, 19. https://doi.org/10.1371/journal.ppat.1000244

Ventura, C.V., Maia, M., Dias, N., Ventura, L.O., and Belfort, R. (2016). Zika: neurological andocular findings in infant without microcephaly. Lancet. 386(10037), 2502. http://dx.doi.org/10.1016 /S0140-67361630776-0

Vogel, G. (2016). Zika virus kills developing brain cells. Science. DOI: 10.1126/Science.aaf4148

World Health Organization [WHO]. (2016, September 6). Zika virus. WHO. [accessed 2017 July]. Retrieved from http://www.who.int/mediacentre/factsheets/zika/en/

Zagorski, J.L. (2016, May 25). How do we know the Zika virus will cost the world $\$ 3.5$ billion? Scientific American. Retrieved from https://www.scientificamerican.com/article/how-dowe-know-the-zika-virus-will-cost-the-world-3-5-billion/ 